\title{
Numerical study on immersed granular collapse in viscous regime by particle-scale simulation
}

Cite as: Phys. Fluids 32, 073313 (2020); https://doi.org/10.1063/5.0015110

Submitted: 26 May 2020 . Accepted: 03 July 2020 . Published Online: 22 July 2020

Yun-hui Sun (孙云辉), Wen-tao Zhang (张炇涛), Xiao-liang Wang (王晓亮), and (D Qing-quan Liu (刘青泉)

\section{COLLECTIONS}

F This paper was selected as Featured
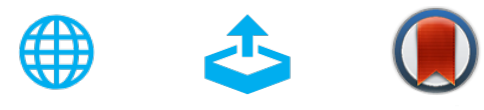

\section{ARTICLES YOU MAY BE INTERESTED IN}

Effects of gravity and surface tension on steady microbubble propagation in asymmetric bifurcating airways

Physics of Fluids 32, 072105 (2020); https://doi.org/10.1063/5.0012796

Investigation and parameterization of transition shielding in roughness-disturbed boundary layer with direct numerical simulations

Physics of Fluids 32, 074110 (2020); https://doi.org/10.1063/5.0012464

Flow-induced fractionation effects on slip of polydisperse polymer melts

Physics of Fluids 32, 073109 (2020); https://doi.org/10.1063/5.0017996

\section{Physics of Fluids GALLERY OF GOVERS}

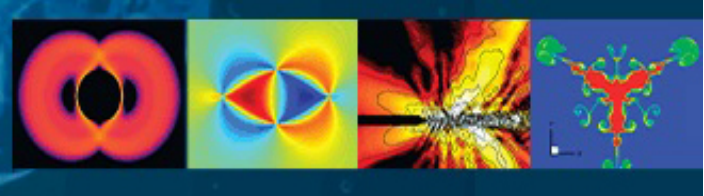




\title{
Numerical study on immersed granular collapse in viscous regime by particle-scale simulation
}

\author{
Cite as: Phys. Fluids 32, 073313 (2020); doi: 10.1063/5.0015110 \\ Submitted: 26 May 2020 - Accepted: 3 July 2020 • \\ Published Online: 22 July 2020
}

Yun-hui Sun (孙云辉), ${ }^{\top}$ Wen-tao Zhang (张炇涛), ${ }^{2}$ Xiao-liang Wang (王晓亮), and Qing-quan Liu (刘青泉) ${ }^{1, a)}$ (1D

\begin{abstract}
AFFILIATIONS
${ }^{1}$ Department of Mechanics, School of Aerospace Engineering, Beijing Institute of Technology, Beijing 100081, China

${ }^{2}$ Key Laboratory for Mechanics in Fluid Solid Coupling Systems, Institute of Mechanics, Chinese Academy of Sciences, Beijing 100190, China
\end{abstract}

a) Author to whom correspondence should be addressed: liuqq@bit.edu.cn

\begin{abstract}
Mixed fluid-particle flows are commonly found in nature and exhibit complex particle-particle and particle-fluid interactions. In this paper, a typical small-scale case of immersed granular collapse under the viscous regime is numerically investigated using computational fluid dynamics coupled with the discrete element method (CFD-DEM), which provide particle-scale information of the collapse. The input parameters for the coupled CFD-DEM model are carefully calibrated from experimental results, and the simulation results achieve good agreement with the experiments in terms of the front evolution and final deposition. The collapse processes for different aspect ratios exhibit similarities and propagate in a three-stage mode that includes acceleration, steady propagation, and deceleration. The propagation velocity, runout distance, and the energy evolution of both fluid and particles are presented. The final runout is linearly proportional to the densimetric Froude number in our high-column cases. The transition of particles' motion from vertical to horizontal and the drag of the fluid are found to be responsible for the constant velocity in the steady propagation stage. We also show that a small energy bump during the initial stage is the result of particle destabilization and rearrangement.
\end{abstract}

Published under license by AIP Publishing. https://doi.org/10.1063/5.0015110

\section{INTRODUCTION}

Large-scale natural disasters such as debris flows, landslides, and submarine avalanches are mostly triggered by earthquakes, structural instabilities, or other unpredictable factors. These destructive events involve the flow of polydispersed granular materials (soil, sand, rock) with interstitial fluid such as water and mud. The presence of a fluid changes the flow dramatically. For example, a submarine landslide may evolve over dozens of miles and even change into turbidity to damage undersea facilities and cables. ${ }^{1}$ Thus, investigating the behavior of mixed fluid-particle flows is important for risk assessments and the design of defense engineering, although the particle-particle and particle-fluid interactions are intrinsically complex.

As a simplified model of fluid-particle mixture flows, smallscale experiments that consider immersed granular collapse have attracted attention in recent years because of their simple configuration and rich dynamics. The two-dimensional dam-break problem has been widely studied. In this kind of experiment, a pile of granular material is initially delimited by a confining gate to form a rectangular block. After the gate is suddenly removed vertically, the granular packing collapses onto a horizontal plane under the action of gravity. Any fluid around the particles is dragged into the motion to form a particle-fluid mixture flow.

Dry granular collapse, i.e., granular collapse in air where the interstitial fluid plays a negligible role, has been investigated since several landmark studies early this century. ${ }^{2-6}$ These investigations demonstrated that the initial aspect ratio of column height $H_{\mathrm{i}}$ to length $L_{\mathrm{i}}$ is the dominant parameter and led to the proposal of simple scaling laws between the final runout $L_{\mathrm{f}}$ and aspect ratio $a=H_{\mathrm{i}} / L_{\mathrm{i}}$, despite the complicated transient flow. Later, Thompson and Huppert ${ }^{7}$ experimentally investigated axisymmetric and two-dimensional collapses in air and compared the results with an underwater collapse. They found that, though the presence of water changes the collapse process significantly, the scaling of the final deposit morphology remains almost the same in both cases. Since then, immersed granular collapse has been widely investigated, both experimentally and numerically. 
Meruane et al. ${ }^{8}$ first developed a two-phase continuum model to study both dry and underwater granular collapses and achieved good agreement with their experimental results for the transient granular surface profile. This work was later extended to deal with dense binary mixture flows. ${ }^{9}$ Rondon et al. ${ }^{10}$ experimentally investigated the influence of the initial volume fraction and aspect ratio and reported a negative pressure under the flow in the case of dense packing and a positive pressure in the case of loose packing. Their experiment is widely used to validate different simulation methods. Savage et al. ${ }^{11}$ used a mixture model to study Rondon's experiment. Though reasonable predictions were achieved for the loose-packing case, the dense-packing case failed essentially because their model neglects the pore pressure effects and the interaction between fluid and particles. Wang et al. ${ }^{12}$ employed a two-fluid continuum theory implemented with smoothed particle hydrodynamics (SPH) to simulate the same problem, with the solid phase modeled as a perfectly elastic-plastic material. Good agreement between the numerical output and the experimental results of Rondon et al. ${ }^{10}$ was observed for the loose case, while for the dense case, the agreement was less satisfactory. They later modified their formula ${ }^{13}$ by determining the dilatancy angle using critical state theory. ${ }^{14}$ Satisfactory agreement between the simulations and their own experiment was then obtained. Si et al. ${ }^{15}$ applied a two-phase model similar to that of Meruane et al. ${ }^{8}$ and obtained good agreement with Rondon's experimental results for both dense packing and loose packing. A two-phase model was also used by Lee and Huang, ${ }^{16}$ who considered turbulent, rheological, and elastic effects. More importantly, they proposed a new particle response time model that combines the Richardson-Zaki model ${ }^{17}$ with Engelund's model ${ }^{18}$ to quantify the drag force under different packing densities. Their simulation of Rondon's experiment produced satisfactory results. They further performed loose-packed experiment combined with numerical simulations focusing on the effect of particle size, where fine particles were shown to have a larger runout distance. ${ }^{19}$

Despite the considerable achievements of continuum methods, their effective application depends on proper modeling of the moment exchange between phases and the closure of the solid stress. In the transient granular collapse problem, the constitutive relationship requires careful consideration of the solid concentration, which plays a significant role in changing the flow behavior and the transition from the granular solid to granular flowing state. ${ }^{10}$ In this regard, discrete methods for granular phase modeling provide an alternative, direct, and powerful tool. Topin et al. ${ }^{20}$ investigated how the initial particle volume fraction and Reynolds number affect the collapse using nonsmooth contact dynamics (NSCD) for particle modeling coupled with a distributed Lagrange multiplier/fictitious domain (DLM/FD). Based on previous research on granular avalanches, ${ }^{21,22}$ they later investigated the collapse dynamics under different flow regimes: grain-inertial (free-fall), fluid-inertial, and viscous. ${ }^{23}$ Despite less horizontal energy being earned by the grains in fluid, the runout for the fluid-inertial regime was almost identical to its dry counterpart (grain-inertial) and longer than for the viscous regime. This phenomenon was attributed to the compensation between friction, viscous, and lubrication effects. A pore-scale simulation in which the lattice Boltzmann method was coupled with discrete element method (LBM-DEM) was used to study pore pressure effects during granular column collapse in two dimensions. ${ }^{24}$
The positive pore pressure development, front water entrainment, and hydroplaning produced a longer runout in the loose case than in the dry case. The simulations conducted by both Topin et al. ${ }^{20,23}$ and Kumar et al. ${ }^{24}$ were implemented in two dimensions to reduce the computational cost, which suffers from the drawback of unreliable pore space. Izard et al..$^{25}$ resolved the fluid in three dimensions using the immersed boundary method coupled with discrete element method (IBM-DEM) and achieved quantitative agreement with the experimental results of Rondon et al., ${ }^{10}$ especially for the basal pore pressure variation. The fluid phase has also been solved using $\mathrm{SPH}^{26}$ which produced a satisfactory comparison with the experimental results of Wang et al. ${ }^{13}$ for the final deposit profile. Recently, a three-dimensional LBM-DEM simulation has been validated and used to analyze the micro-mechanical mechanism of a quasi-two-dimensional immersed granular collapse. ${ }^{27,28}$ These discrete simulations gave reasonable results, but were generally computationally expensive and time consuming because the fluid resolution must be smaller than the particle spacing to resolve the fluid flow at the pore scale. Thus, the number of discrete particles is limited to several thousand (Table I), which restricts their wider applications. This paper studies granular flows with much more particles, so it requires more consideration for a proper simulation method.

Of particular interest is the work of Jing et al., ${ }^{29}$ who studied immersed granular collapse using computational fluid dynamics (CFD) and DEM for the particle (CFD-DEM). This method requires a coarse fluid grid in which the cells are larger than the particle diameter to ensure a reasonable averaged continuum field and achieves coupling through particle-fluid interaction force models. It has the advantage of relatively low computational cost compared to the aforementioned resolved methods and has greater capacity to capture the particle-scale motion compared with two-fluid continuum methods. ${ }^{30}$ Note that Jing et al. ${ }^{29}$ did not reproduce the details of any existing experiments, as this requires careful parameter calibration. Instead, they mainly focused on the influence of the aspect ratio and different flow regimes. ${ }^{31}$ To the best of the authors' knowledge, there have been few detailed calibrations of granular parameters from experiments in the study of immersed granular collapse. Therefore, the main goal of this study is to reproduce immersed granular collapse through CFD-DEM simulations and provide a deeper view of the collapse procedure. We restrict this study to granular collapse in

TABLE I. Previous discrete-continuum methods used to study immersed granular collapse. $d_{p}, \varepsilon_{s}, N_{\text {par }}^{\max }$ denote particle diameter, solid volume fraction, and maximum number of particles used, respectively.

\begin{tabular}{|c|c|c|c|c|}
\hline Investigator & Methodology & $d_{\mathrm{p}}(\mathrm{mm})$ & $\mathcal{E}_{\mathrm{s}}$ & $N_{\text {par }}^{\max }$ \\
\hline Topin et al. ${ }^{23}$ & 2D CD-DLM/FD & 1 & 0.8 & 1450 \\
\hline Kumar et al. ${ }^{24}$ & 2D LBM-DEM & $\ldots$ & $0.79,0.83$ & 1000 \\
\hline Izard et al. ${ }^{25}$ & IBM-DEM & 15.2 & $0.58,0.60$ & $\approx 1000^{\mathrm{a}}$ \\
\hline Jing et al. ${ }^{29}$ & CFD-DEM & 1 & 0.56 & $\approx 34000^{\mathrm{a}}$ \\
\hline $\mathrm{Xu}$ et $a l^{26}$ & SPH-DEM & $0.5-0.6$ & 0.49 & 27030 \\
\hline Yang et $a l^{27}$ & LBM-DEM & 1.436 & $0.55-0.63$ & $\approx 5000^{\mathrm{a}}$ \\
\hline
\end{tabular}

${ }^{\mathrm{a}} N_{\mathrm{par}}^{\max }$ is calculated as $V_{\text {total }} \varepsilon_{\mathrm{s}} / V_{0}$, where $V_{0}$ is the particle volume. 
the viscous regime ${ }^{32}$ and perform detailed calibration of the model parameters.

The remainder of this paper is organized as follows: Section II introduces the methodology of the coupled CFD-DEM simulations, which use well-determined parameters. The simulation setup is presented in Sec. III. Simulation results of immersed granular collapse are compared with experimental results in Sec. IV, allowing the collapse dynamics to be further analyzed. Finally, Sec. V summarizes the conclusions from this study.

\section{NUMERICAL METHODOLOGY}

\section{A. CFD-DEM formulation}

The approach of combining CFD with DEM is widely used to investigate coupled fluid-particle flows. ${ }^{33,34}$ In this method, the particles are modeled by $\mathrm{DEM}^{35}$ based on Newton's second law and the fluid flow is solved by traditional CFD according to the volume-averaged Navier-Stokes equations, similar to those used in two-phase models (TPMs). Their coupling is achieved by interaction forces, which exchange momentum between phases. ${ }^{36}$

The CFD part solves the volume-averaged incompressible Navier-Stokes equations, which are written in the following form: ${ }^{36,37}$

$$
\begin{gathered}
\partial \varepsilon_{\mathrm{f}} / \partial t+\nabla \cdot\left(\varepsilon_{\mathrm{f}} \boldsymbol{u}_{\mathrm{f}}\right)=0, \\
\partial\left(\varepsilon_{\mathrm{f}} \boldsymbol{u}_{\mathrm{f}}\right) / \partial t+\nabla \cdot\left(\varepsilon_{\mathrm{f}} \boldsymbol{u}_{\mathrm{f}} \boldsymbol{u}_{\mathrm{f}}\right)=-\varepsilon_{\mathrm{f}} \nabla p / \rho_{\mathrm{f}}+\nabla \cdot\left(\varepsilon_{\mathrm{f}} \boldsymbol{\tau}\right) / \rho_{\mathrm{f}}-\boldsymbol{F}_{\mathrm{pf}} / \rho_{\mathrm{f}},
\end{gathered}
$$

where $\mathrm{u}, \varepsilon_{\mathrm{f}}, \rho_{\mathrm{f}}, p$ denote the fluid velocity, porosity, density, and pressure, respectively; $\boldsymbol{\tau}=\mu_{\mathrm{f}}\left(\nabla \boldsymbol{u}_{f}+\nabla \boldsymbol{u}_{f}^{\mathrm{T}}\right)$ is the deviatoric stress tensor of the fluid, with $\mu_{\mathrm{f}}$ being the dynamic viscosity. The interaction force acting by the granular phase is given by

$$
\boldsymbol{F}_{\mathrm{pf}}=\sum_{i=1}^{n} \boldsymbol{f}_{\mathrm{d}, i} / \Delta \boldsymbol{V}
$$

where $\boldsymbol{f}_{\mathrm{d}, i}$ is the drag force and $\Delta \boldsymbol{V}$ is the cell volume. Other interaction forces, such as the virtual mass force, lift force, and history force, are neglected in this study because they are insignificant compared with the drag force. ${ }^{15}$

The transitional and rotational motions of particles are governed by Newton's second law according to $m_{i} \frac{\mathrm{d} \boldsymbol{u}_{\mathrm{p}, i}}{\mathrm{~d} t}=\sum_{j}\left(\boldsymbol{f}_{i j}^{\mathrm{c}, \mathrm{n}}+\boldsymbol{f}_{i j}^{\mathrm{c}, \mathrm{t}}\right)+m_{i} \boldsymbol{g}+\boldsymbol{f}_{\nabla p, i}+\boldsymbol{f}_{\nabla \cdot \tau, i}+\boldsymbol{f}_{\mathrm{buo}, i}+\boldsymbol{f}_{\text {lubri }, i}+\boldsymbol{f}_{\mathrm{d}, i}$,

$$
I_{i} \frac{\mathrm{d} \boldsymbol{\omega}_{\mathrm{p}, i}}{\mathrm{~d} t}=\sum_{j}\left(\boldsymbol{M}_{\mathrm{t}, i j}+\boldsymbol{M}_{\mathrm{r}, i j}\right),
$$

where $\boldsymbol{u}_{\mathrm{p}, i}$ and $\boldsymbol{\omega}_{\mathrm{p}, i}$ are the $i$-th particle's transitional and rotational velocities, respectively. The forces on the right-hand side of Eq. (3) include contact force $\boldsymbol{f}_{i j}^{c, n}$ and $\boldsymbol{f}_{i j}^{c, t}$, gravity $m_{i} \boldsymbol{g}$, pressure gradient force $\boldsymbol{f}_{\nabla p, i}$, viscous force $\boldsymbol{f}_{\nabla \cdot \boldsymbol{\tau}, i}$, buoyant force $\boldsymbol{f}_{\text {buo }, i}$, lubrication force $f_{\text {lubri, } i}$ and drag force $f_{\mathrm{d}, i}$. The normal and tangential components of the contact force of particle $j$ on particle $i$ are given by

$$
\begin{aligned}
& \boldsymbol{f}_{i j}^{\mathrm{c}, \mathrm{n}}=\left(k_{\mathrm{n}} \delta_{\mathrm{n}}-\gamma_{\mathrm{n}} u_{\mathrm{n}}\right) \boldsymbol{n}_{i j}, \\
& \boldsymbol{f}_{i j}^{\mathrm{c}, \mathrm{t}}=\min \left(k_{\mathrm{t}} \delta_{\mathrm{t}}-\gamma_{\mathrm{t}} u_{\mathrm{t}}, \mu_{\mathrm{p}-\mathrm{p}} f_{i j}^{\mathrm{c}, \mathrm{n}}\right) \boldsymbol{t}_{i j},
\end{aligned}
$$

where the labels $\mathrm{n}$ and $\mathrm{t}$ denote normal and tangential components, respectively. The spring coefficient $k$ and damping coefficient $\gamma$ are calculated by the nonlinear Hertz model. ${ }^{38}$ The coefficient of internal friction $\mu_{\mathrm{p}-\mathrm{p}}$ is equal to $\tan \theta_{\mathrm{r}}$, where $\theta_{\mathrm{r}}$ is the repose angle of the granular material.

The last five terms in Eq. (3) are the forces induced by the fluid. The pressure gradient force $\boldsymbol{f}_{\nabla p, i}=V_{i} \nabla p\left(V_{i}=\pi d_{\mathrm{p}}^{3} / 6\right.$ being the particle volume) and viscous force $\boldsymbol{f}_{\nabla \cdot \tau, i}=V_{i} \nabla \cdot \boldsymbol{\tau}$ are due to macroscopic variation of fluid stress tensor, which comes from the formulation stage. Different from viscous force, the drag force $\boldsymbol{f}_{\mathrm{d}, i}$ comes from the locally relative motion between fluid and particles, which induces shear stress on the particles considering the non-slip condition. There are many drag force models. ${ }^{39,40}$ Table II briefly summarizes several drag force models used in previous immersed granular collapse studies. This study employs the drag force model used by Lee and Huang, ${ }^{16}$ who combined the Richardson-Zaki (RZ) and Engelund models, because this gives the best results. The drag force model can be written as

$$
\boldsymbol{f}_{\mathrm{d}, i}=\varepsilon_{\mathrm{f}} \rho_{\mathrm{s}} V_{i}\left(\boldsymbol{u}_{\mathrm{f}}-\boldsymbol{u}_{\mathrm{p}}\right) / \tau_{\mathrm{p}}
$$

where $\tau_{\mathrm{p}}$ is the response time, details of which can be found in

\begin{tabular}{|c|c|c|c|c|c|}
\hline Investigator & Methodology & $d_{\mathrm{p}}(\mathrm{mm})$ & $\varepsilon_{\mathrm{s}}$ & $\mu_{\mathrm{f}}(\mathrm{cp})$ & Drag model \\
\hline Meruane et al. ${ }^{8}$ & TPM & $0.2,0.7,3$ & 0.60 & $0.02,1$ & Di Felice \\
\hline Wang et al..$^{12}$ & TPM (SPH) & 0.225 & $0.55,0.60$ & 12 & Darcy's law \\
\hline Wang et al. ${ }^{13}$ & EXP\&TPM & 0.3 & $0.55,0.60$ & 1,12 & Darcy's law \\
\hline Lee and Huang ${ }^{16}$ & TPM & 0.225 & $0.55,0.58$ & 12 & RZ-Engelund \\
\hline Lee et al. ${ }^{19}$ & EXP\&TPM & $0.12,0.56$ & 0.55 & 1 & $\mathrm{RZ}$ \\
\hline Si et al. ${ }^{15}$ & TPM & 0.225 & $0.55,0.60$ & 1 & Gidaspow \\
\hline Jing et al. ${ }^{29}$ & CFD-DEM & 1 & 0.56 & 1 & Di Felice \\
\hline This paper & CFD-DEM & 3.84 & 0.64 & 29.7 & RZ-Engelund \\
\hline
\end{tabular}
Ref. 16.

The lubrication force is from radial pressure of fluid when two particles get close and the interstitial fluid is squeezed out. Since we have a coarse mesh larger than the particles and the lubrication effect cannot be resolved with the computational mesh, the

TABLE II. Drag force models used in previous studies on immersed granular collapse. EXP denotes experiment. 
lubrication force is added explicitly in Eq. (3) based on lubrication theory ${ }^{41}$ as

$$
f_{\text {lubri, } i}=6 \pi \mu R_{\mathrm{eff}}^{2} \boldsymbol{v}_{\mathrm{rel}}^{\mathrm{n}} / \delta_{\mathrm{n}}\left(0.001 R_{\mathrm{eff}}<\delta_{\mathrm{n}}<R_{\mathrm{eff}}\right),
$$

where $R_{\mathrm{eff}}=R_{i} R_{j} /\left(R_{i}+R_{j}\right)$ is the effective radius of particles $i$ and $j$, which have radii $R_{i}$ and $R_{j}$, respectively.

Noting that we are considering incompressible fluid and we do not add gravity in the fluid's governing equation, i.e., Eq. (1), the fluid pressure thus does not contain the hydrostatic part, which should have induced buoyant force considering the pressure gradient force $\left(f_{\nabla p, i}=V_{i} \nabla p\right)$ acting on the particles. So we also need to add buoyant force explicitly in Eq. (3) as $\boldsymbol{f}_{\text {buo }, i}=-V_{i} \rho_{\mathrm{f}} \boldsymbol{g}$.

In Eq. (4), $\boldsymbol{M}_{\mathrm{t}, i j}$ is the contact torque from the tangential force and $\boldsymbol{M}_{\mathrm{r}, i j}$ is the rolling resistance, which prevents free rolling and accounts for the shape effect of nonspherical particles. ${ }^{42}$ The elasticplastic spring-dashpot (EPSD) model for rolling friction is applied here: ${ }^{43}$

$$
\boldsymbol{M}_{\mathrm{r}, i j}^{t+\Delta t}=\min \left(\boldsymbol{M}_{\mathrm{r}, i j}^{t}-2.25 k_{\mathrm{n}} \mu_{\mathrm{r}}^{2} R_{\mathrm{eff}}^{2} \Delta \theta_{\mathrm{r}}, \mu_{\mathrm{r}} R_{\mathrm{eff}} f_{i j}^{\mathrm{c}, \mathrm{n}}\right) .
$$

$\Delta \theta_{\mathrm{r}}$ is the incremental relative rotation between particles. The coefficient of rolling friction $\mu_{\mathrm{r}}$ is to be calibrated.

For the implementation of this CFD-DEM formulation, we make use of the open-source software package of CFDEM ${ }^{\circledR 44}$ which combines the DEM code LIGGHTS ${ }^{\circledR 36}$ and CFD code OpenFOAM $^{\circledR}$. The drag force and the lubrication force are implemented with $\mathrm{C}++$ coding. The pressure implicit with splitting of operators (PISO) algorithm is used for fluid solver. A divided porosity model in $\mathrm{CFDEM}^{\circledR}$ is used to map particles' volume to porosity field. The time discretization is in implicit Euler scheme and spatial discretization in linear interpolation.

\section{B. Calibration of simulation parameters}

In dense granular flows, the contacts among particles dominate than other hydrodynamic or lubrication forces. ${ }^{45}$ In this research, we intend to reproduce experimental results through CFD-DEM simulations. Thus, proper calibration of the granular parameters is a significant task and is mainly achieved by laboratory tests.

Young's modulus $E$ and Poisson's ratio $\sigma$ of borosilicate glass, which are required by Hertz's model to calculate the contact forces, are $63 \mathrm{GPa}$ and 0.2 , respectively, according to the manufacturer. Such a high modulus implies a very short DEM time step, so we decrease its value by three orders of magnitude to $63 \mathrm{MPa}$. This does not have much influence on the granular flow, ${ }^{42,46}$ but reduces the computational cost significantly by increasing the required DEM time step.

The coefficient of friction between the particles and walls $\mu_{\mathrm{p}-\mathrm{w}}$ is determined by a slide test. First, a thin cylindrical tube is filled with particles to form a direct contact face between the particles and a glass plate (same material as the water tank), as shown in Fig. 1(a). The plate is then slowly tilted. The angle at which the cylinder starts to slide, denoted by $\theta_{\text {slide, }}$ is measured by a clinometer, and we have $\mu_{\mathrm{p}-\mathrm{w}}=\tan \theta_{\text {slide }}$.

The repose angle is measured by slowly pouring a known amount of beads from a funnel onto a granular pile from above. As shown in Fig. 1(b), the edge profile is extracted by image processing and linearly interpolated to give the slope, which
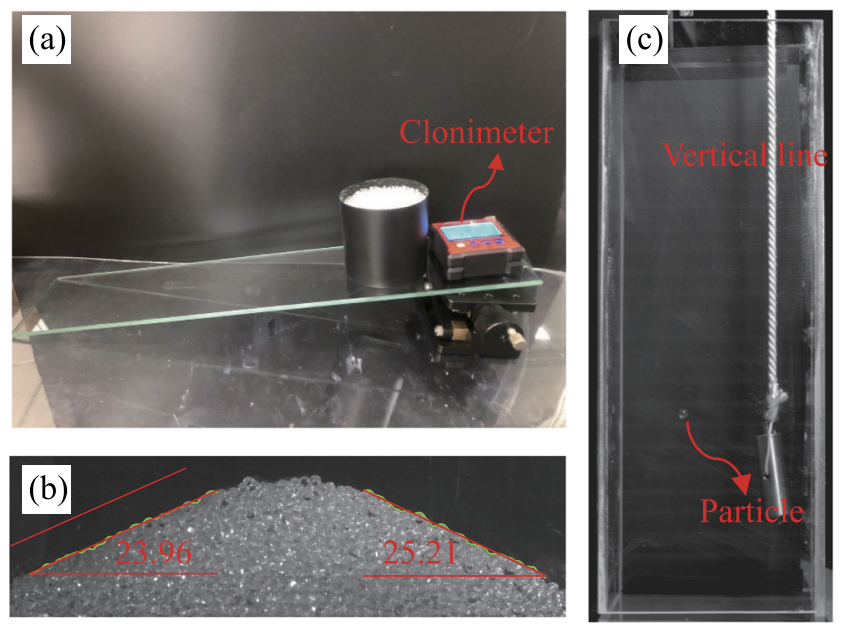

FIG. 1. Measurement of model parameters: (a) Friction coefficient between particles and glass plate. (b) Repose angle. (c) Coefficient of restitution between particle and glass plate.

is considered to be the repose angle. ${ }^{47}$ Pictures from different directions are processed, and the averaged angle is finally defined as the repose angle $\theta_{\mathrm{r}}$. We also use this simple experiment to calibrate the coefficient of rolling friction $\mu_{\mathrm{r}}$ by trial and error. The same procedure is simulated by pure DEM simulation, and the final deposits are postprocessed using the same method as for the experiment. A value of $\mu_{\mathrm{r}}=0.035$ is found to produce the closest repose angle to the experiment, so this value is adopted in subsequent simulations.

The coefficient of restitution between the particle and the wall, denoted as $e_{\mathrm{p}-\mathrm{w}}$, is determined by a drop test, ${ }^{48}$ as shown in Fig. 1(c). A particle is dropped from a given height $h$, and the maximum height $h^{\prime}$ reached after one bounce is recorded by a high-speed camera. The coefficient of restitution is calculated according to the energy lost as $e_{\mathrm{p}-\mathrm{w}}=\sqrt{h^{\prime} / h}$, and the coefficient of restitution between particles $e_{\mathrm{p}-\mathrm{p}}$ is considered to be equal to $e_{\mathrm{p}-\mathrm{w}}$, i.e., $e_{\mathrm{p}-\mathrm{w}}$ $=e_{\mathrm{p}-\mathrm{p}}=e$.

\section{Verification of granular parameters}

We now verify the input parameters calibrated in Sec. II B through a dry granular collapse test with an initial aspect ratio of $a=2$. The simulated collapse sequence is shown in Figs. 2(a)-2(c), in which only particles near the front wall are presented. Ignoring those indistinct particles at the back because the camera is focusing on the front wall, ${ }^{49}$ the collapse process for the dry case is successfully reproduced, implying that the granular parameters are correctly calibrated.

It should be emphasized that we apply a gate-removal process in the simulations, and gate-particle interaction is regarded the same as wall-particle interactions. The trajectory of the gate's lower edge is presented in Fig. 2(d), where rectangular markers indicate raw data extracted from the experimental images and the solid line represents the quadratic polynomial fitting result. Good agreement between the raw and fitted data indicates that the gate is uniformly accelerating. 

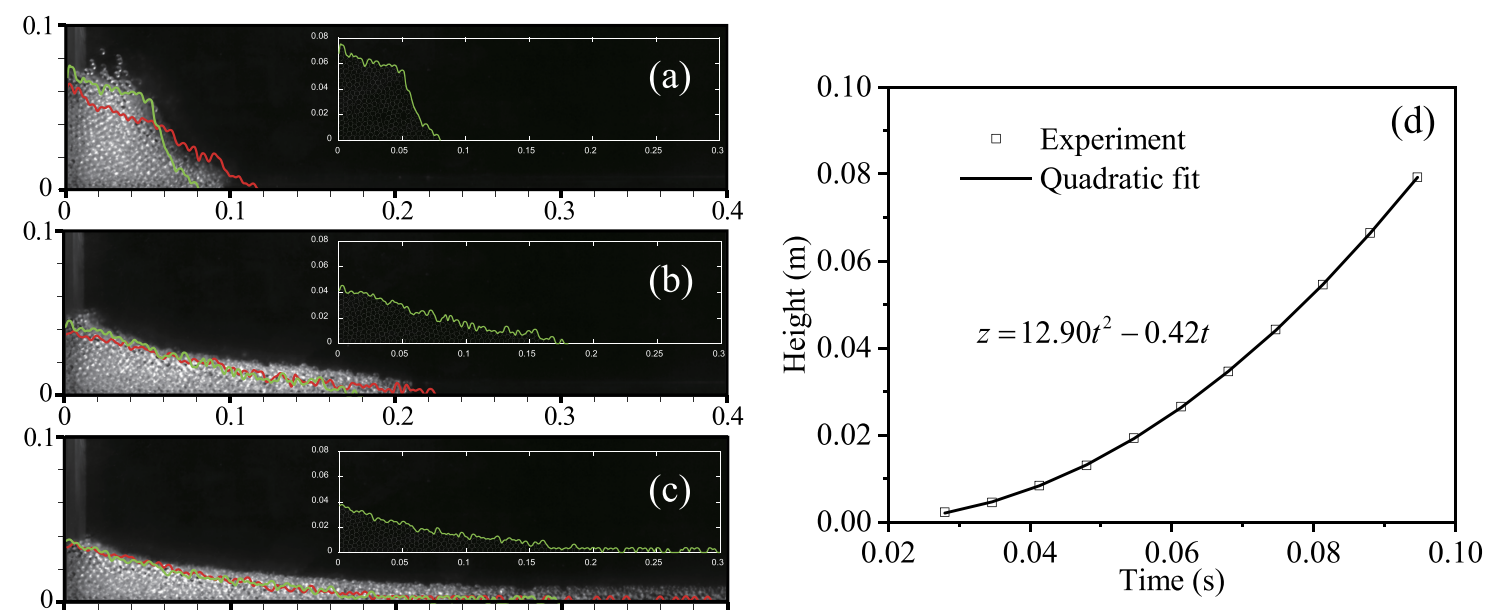

FIG. 2. Dry granular collapse for $a=2:[(a)-(c)]$ Comparison of granular collapse profile at $t=0.15 \mathrm{~s}, t=0.30 \mathrm{~s}$, and $t=1.00 \mathrm{~s}$, respectively. The insets illustrate the simulated results with gate removal and their upper surface profiles (not to scale). Red lines refer to simulation without gate removal. (d) Gate-removal trajectory.

\section{SIMULATION OF IMMERSED GRANULAR COLLAPSE}

\section{A. Experimental results for comparison with simulations}

The immersed granular collapse we attempt to simulate here was conducted in a $40 \times 8 \times 30 \mathrm{~cm}^{3}$ glass water tank, as shown in Fig. 3(a). The particles are borosilicate glass beads $\left(d_{\mathrm{p}}=3.84\right.$ $\pm 0.3 \mathrm{~mm}$ ) with a measured density of $2178 \mathrm{~kg} / \mathrm{m}^{3}$. A mixture of equal mass fractions of dimethyl sulfoxide aqueous solution and glycerin aqueous solution is used as the fluid, giving a dynamic viscosity of $\mu_{\mathrm{f}}=29.1 \mathrm{cp}$ and a density of $\rho_{\mathrm{f}}=1167 \mathrm{~kg} / \mathrm{m}^{3}$.

In the experiment, the glass tank is filled with the well-mixed fluid and a rectangular zone is delimited by a 3-mm-thick stainlesssteel gate at $x=L_{\mathrm{i}}$. We fix $L_{\mathrm{i}}=4 \mathrm{~cm}$ and vary the column height $H_{\mathrm{i}}$ to form different aspect ratios $a=[2-6]$. The initial solid volume fraction $\varepsilon_{s}$ is around 0.64 . To visualize the glass beads, as shown in Fig. 3(b), a blue laser sheet is shot along the bottom wall from the front and a mirror is placed under the tank at a $45^{\circ}$ angle to the horizontal plane. Once the gate is lifted up, a camera is going to record the collapse process. We also record the final deposit from the front after the collapse. Typical images obtained are shown in Figs. 3(c) and $3(\mathrm{~d})$.

For each aspect ratio, the experiment is repeated three times. The experimental pictures observed from the bottom are processed to extract the evolution of the leading front with respect to time, denoted as $L_{\mathrm{t}}$. The experimental results for the front evolution possess good repeatability, as shown in Fig. 4.

\section{B. Simulation setup for immersed granular collapse}

We adopt homogeneous $40 \times 8 \times 30$ mesh with size of $0.01 \mathrm{~m}$, which is about $2.6 d_{\mathrm{p}}$. The number of particles is
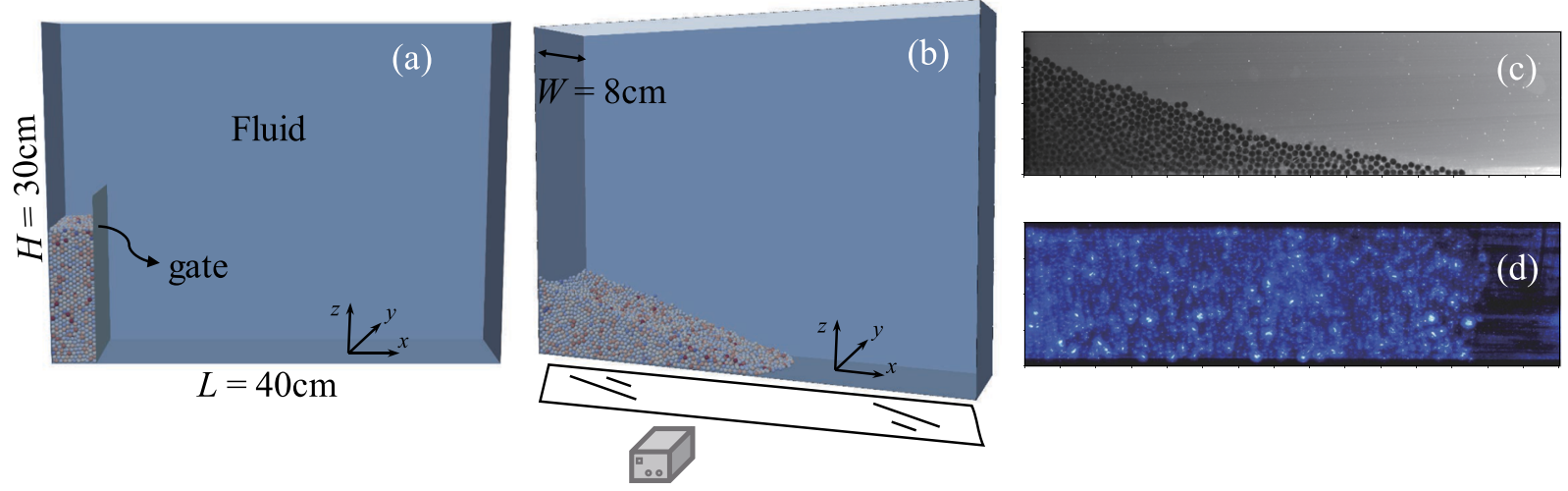

FIG. 3. Configuration of immersed granular collapse: (a) Initial column. (b) Final deposit. A mirror is placed below the tank to observe particulate bottom evolution. Raw experimental image shot from front (c), where particles appear black, and bottom (d), where particles appear blue. 


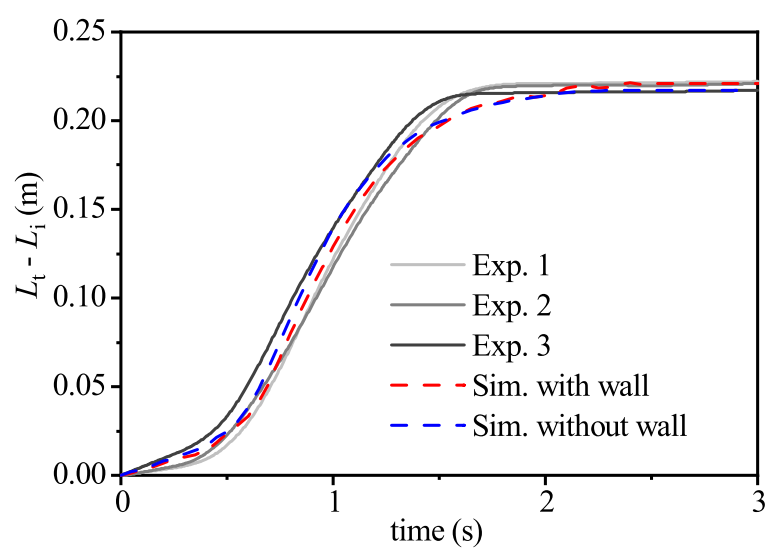

FIG. 4. Front evolution of three repeated experiments for $a=4$. Simulation results given by CFD-DEM are also presented.

aspect-ratio-dependent as 2756a. Detailed modeling parameters for the CFD-DEM simulations are presented in Table III. Despite the well-determined granular parameters, there are still some setup questions that are worthy of discussion, including the effects of the procedure of removing the gate and the boundary conditions.

\section{Gate-removal effects}

The movement of the confining gate, which triggers the collapse in the experiments, does not actually exist in natural collapse events. However, in our experiments, the moving gate influences the collapse significantly by inducing initial elevation of the granular pile and vertical fluid velocity. Several studies on this problem have concluded that the influence only exists in the initial stage. ${ }^{12,13,15,16,19}$ In our simulation of the dry case, the gate-removal stage lasts about $0.1 \mathrm{~s}$, which is approximately one-tenth of the collapse duration. The foot of the granular packing runs almost twice as far without a wall during the initial stage [see red lines in Fig. 2(a)]. In the following simulation of immersed cases, we apply a moving gate only to the particles, i.e., the DEM part, while no such setting for the CFD part. The gate-removal stage lasts no more than $5 \%$ of the collapse process, and no induced fluid velocity is applied. The result suggests that this manipulation does not affect the collapse process as much as in dry cases (see the blue dashed line in Fig. 4). Regardless, the gate-removal process is adopted in this study for completeness and further discussion. As will be seen later, the gateremoval process can suppress the energy bump appearing during the initial stage.

\section{Wall effects and boundary conditions}

The fluid used in our experiments has a viscosity of 29.7 $\mathrm{cp}$, which is higher than in most previous experiments or simulations on submarine granular collapse. Higher viscosity means a thicker boundary layer in which the fluid velocity is slow and the particulate motion is retarded. Previous simulations were mainly performed with TPMs or discrete-element-based methods. The former are mainly implemented in two dimensions, $8,11-13,15,16,19,26$ whereas the latter are either two-dimensional ${ }^{20,23,24}$ or quasi-twodimensional $^{28,29,31}$ through the application of periodic boundary conditions in the spanwise direction. We perform unresolved CFDDEM simulations that require a coarse mesh in which the cells are larger than the particles to ensure the reasonable calculation of the volume fraction field and particle-fluid moment exchange, i.e., Eq. (2). The coarse mesh prevents us from accurately calculating both the fluid flow and particle motion near the side wall, though there are several methods to solve this problem ${ }^{50,51}$ that deserve further investigation. This is why we only compare particle motion from the bottom in this study, because the granular front is in the inner region where wall effects are minor. The boundary layer effects near the bottom are believed to be insignificant, as the bottom particles are mainly pushed into motion by upper particles. Furthermore, a periodic boundary condition has been considered and the collapse runs much longer for higher columns, such that the periodic boundary condition is not adopted here. In this study,

TABLE III. Model parameters used for CFD-DEM simulations of immersed granular collapse.

\begin{tabular}{llc}
\hline \hline \multicolumn{1}{c}{ Parameters } & Value \\
\hline Simulation region & Length & $40 \mathrm{~cm}$ \\
& Height & $30 \mathrm{~cm}$ \\
Particle & Width & $8 \mathrm{~cm}$ \\
& Diameter, $\rho_{\mathrm{p}}$ & $3.84 \pm 0.3 \mathrm{~mm}$ \\
& Particle density, $\rho_{\mathrm{p}}$ & $2178 \mathrm{~kg} / \mathrm{m}^{3}$ \\
& Young's modulus, $E$ & $6.3 \times 10^{7} \mathrm{~Pa}$ \\
& Poisson's ratio, $\sigma$ & 0.2 \\
& Coefficient of restitution, $e$ & 0.91 \\
& Coefficient of friction between particles and wall $\mu_{\mathrm{p}-\mathrm{w}}$ & 0.19 \\
Fluid & Coefficient of friction between particles $\mu_{\mathrm{p}-\mathrm{p}}$ & 0.47 \\
& Coefficient of rolling friction $\mu_{\mathrm{r}}$ & 0.035 \\
Drag model & Fluid density, $\rho_{\mathrm{f}}$ & $1167 \mathrm{~kg} / \mathrm{m}^{3}$ \\
& Fluid kinetic viscosity, $v_{\mathrm{f}}$ & $2.49 \times 10^{-5} \mathrm{~m}^{2} \mathrm{~s}^{-1}$ \\
\hline \hline
\end{tabular}


a zero-pressure condition is applied on the upper boundary and noslip conditions for velocity are imposed on the other boundaries. A zero-gradient boundary condition is applied as the default for the other parameters.

\section{Simulation procedure}

The simulations are conducted according to the following procedure:

(1) Preparation: Particles are generated randomly in a region under reduced gravity: $g^{\prime}=\left(1-\rho_{\mathrm{f}} / \rho_{\mathrm{p}}\right) g$ with $g=9.81 \mathrm{~m} \mathrm{~s}^{-2}$. To produce the packing density (0.64) of the experiments, the generation region is slightly higher than the target region and the particles have quite a low friction coefficient (0.051) and restitution coefficient (0). A moving wall is applied from above to press the particles down to $H^{\prime}=0.9 a L_{\mathrm{i}}$ and then retracted.

(2) Relaxation: The friction coefficient and restitution coefficient are adjusted to their correct values. After relaxation, the wellprepared initial pile is passed to the CFD-DEM solver and the simulation runs under full gravity with the lateral gate fixed to relax any possible movement. The final height of the column is found to be around $a L_{\mathrm{i}}$ (no more than half a particle diameter higher).

(3) Release: The gate then moves vertically following the calibrated trajectory, and the granular collapse begins under the self-weight of the particles. The moment the gate starts moving is regarded as the beginning of the collapse process, i.e., $t=0$.

\section{RESULTS AND DISCUSSION}

\section{A. Comparison between simulation and experimental results}

The front evolution in the simulation results is extracted based on particle positions, with only particles near the bottom taken into account. There is good agreement between the simulated and experimental results for the front evolution, as shown in Fig. 5(a). The red dashed line in Fig. 4 shows that the simulation result is within the normal range of experimental error.

We further normalize the travel distance according to $H_{\mathrm{i}}$ and time according to $\tau_{\mathrm{c}}=\sqrt{H_{\mathrm{i}} / g^{\prime}}$. This scaling is known to be appropriate, ${ }^{29}$ and the normalized evolutions with different aspect ratios are almost identical in Fig. 5(b), except for the final runout. Similar to previous studies on granular collapse, our results clearly show early acceleration, followed by steady propagation (constant velocity), and then deceleration. In Fig. 5(b), it is remarkable that the constant velocity stage occurs from $4 \tau_{c}-6 \tau_{c}$, despite the different aspect ratios.

Though we do not intend to reproduce the instantaneous granular pile profile during the collapse process, the final deposit profiles are in satisfactory agreement with the experimental results, as shown in Fig. 6 for exemplar cases of $a=2,4$, and 6 .
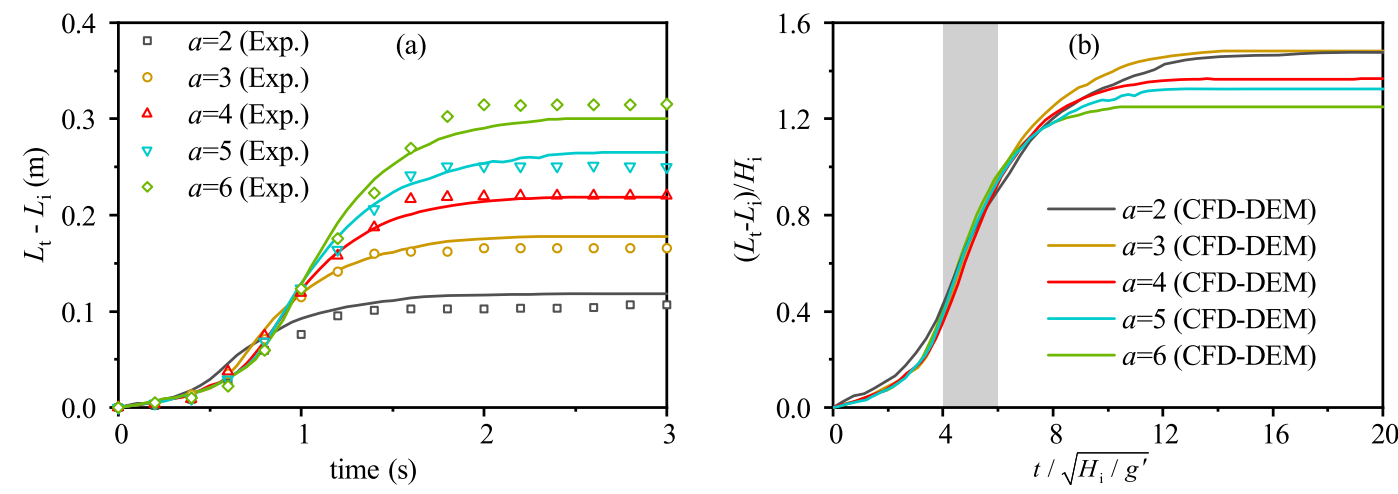

FIG. 5. Front evolution with different aspect ratios. (a) Comparison between CFD-DEM results and experimental results. (b) Normalized front evolution. The gray part indicates the steady propagation stage.

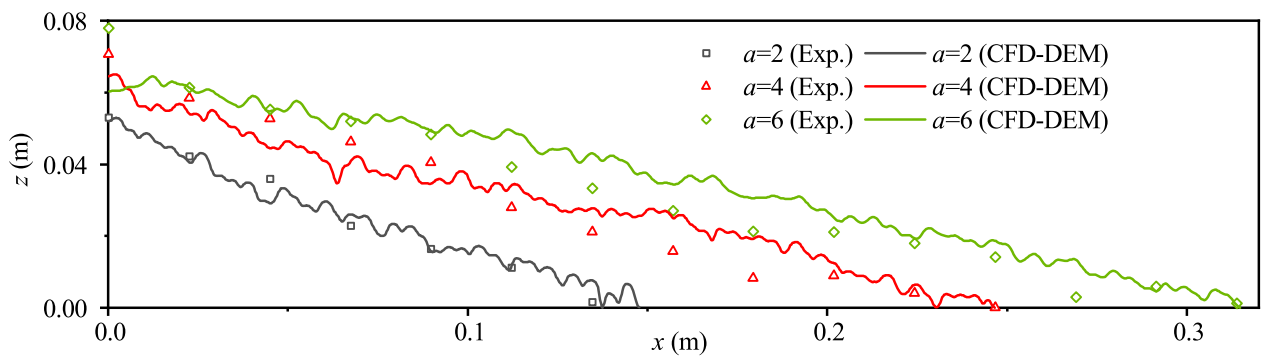

FIG. 6. Comparison of final deposit profile between experimental and simulation results for cases $a=2,4$, and 6 . 


\section{B. Collapse process}

Figure 7 presents collapse snapshots at four sequential times with $a=4$. Only the central slice of fluid at $y=4 \mathrm{~cm}$ and particles near the central slice $(y=4 \pm 0.5 \mathrm{~cm})$ are shown, where the wall effect is minor. At $t=0.2 \mathrm{~s}$, the bottom particles have just traveled one particle diameter out of the initial state (see the white profile). This time can also be defined as the trigger time, ${ }^{32}$ after which the collapse evolves dramatically. Particles near the right-upper corner move slightly faster and quickly fall down to the bottom, forming one large vortex as shown in Fig. 7(b). The steady propagation stage starts at $0.75 \mathrm{~s}\left(\approx 4 \tau_{\mathrm{c}}\right)$. It can be observed that the corner particles remain almost static and an inclined shear plane is formed. Particles that are initially in the right portion of the domain mix together to form the granular front and evolve at a constant velocity as the vortex moves down. After $1.12 \mathrm{~s}\left(\approx 6 \tau_{\mathrm{c}}\right)$, the collapse starts to decelerate and falling particles gradually approach their final height. At $t=1.7 \mathrm{~s}$, the particles behind the front have almost stopped. The vortex gradually fades, and the existing flow drives the front particles forward to their final position.

\section{Propagation velocity}

For dense particle-fluid flows, there exist three regimes: viscous, inertial, and free-fall, depending on the properties of both the particles and the ambient fluid. ${ }^{22}$ Such flows can be represented by two dimensionless numbers, the Stokes number St and density ratio $r$, given by

$$
\mathrm{St}=\frac{\sqrt{\rho_{\mathrm{p}}\left(\rho_{\mathrm{p}}-\rho_{\mathrm{f}}\right) g d_{\mathrm{p}}^{3}}}{18 \mu_{\mathrm{f}}}, \quad r=\sqrt{\frac{\rho_{\mathrm{p}}}{\rho_{\mathrm{f}}}} .
$$

Recently, Bougouin and Lacaze ${ }^{32}$ systematically studied immersed granular collapse in different regimes through a series of experiments. The maximum front velocity was found to be linearly dependent on the free-fall velocity,

$$
U=\zeta(\mathrm{St}) U_{\mathrm{FF}}, \text { where } U_{\mathrm{FF}}=\sqrt{2 g^{\prime} H_{\mathrm{i}}} .
$$

The coefficient $\zeta$ is St-dependent, but their experiments did not determine whether $\zeta$ depends on the density ratio because $r$ hardly varied. In this study, Eq. (9) gives St $=1.46$ and $r=1.37$, which can be compared with the viscous inertial regime cases in Bougouin and Lacaze, ${ }^{32}$ where $\mathrm{St}=1.5$ and $r=1.6$. The St values are similar, whereas the density ratio $r$ is slightly different. Here, the propagation velocity $U$ is obtained by linearly fitting the front position during the constant velocity stage, and the results exhibit perfect goodness of fit for all aspect ratios $\left(\mathrm{R}^{2}>0.99\right)$.

Figure 8 shows the normalized front propagation velocity. The velocity normalized by the free-fall velocity $\zeta=U / U_{\mathrm{FF}}$ is located well within the error bar (gray area) obtained by Bougouin and Lacaze. ${ }^{32}$
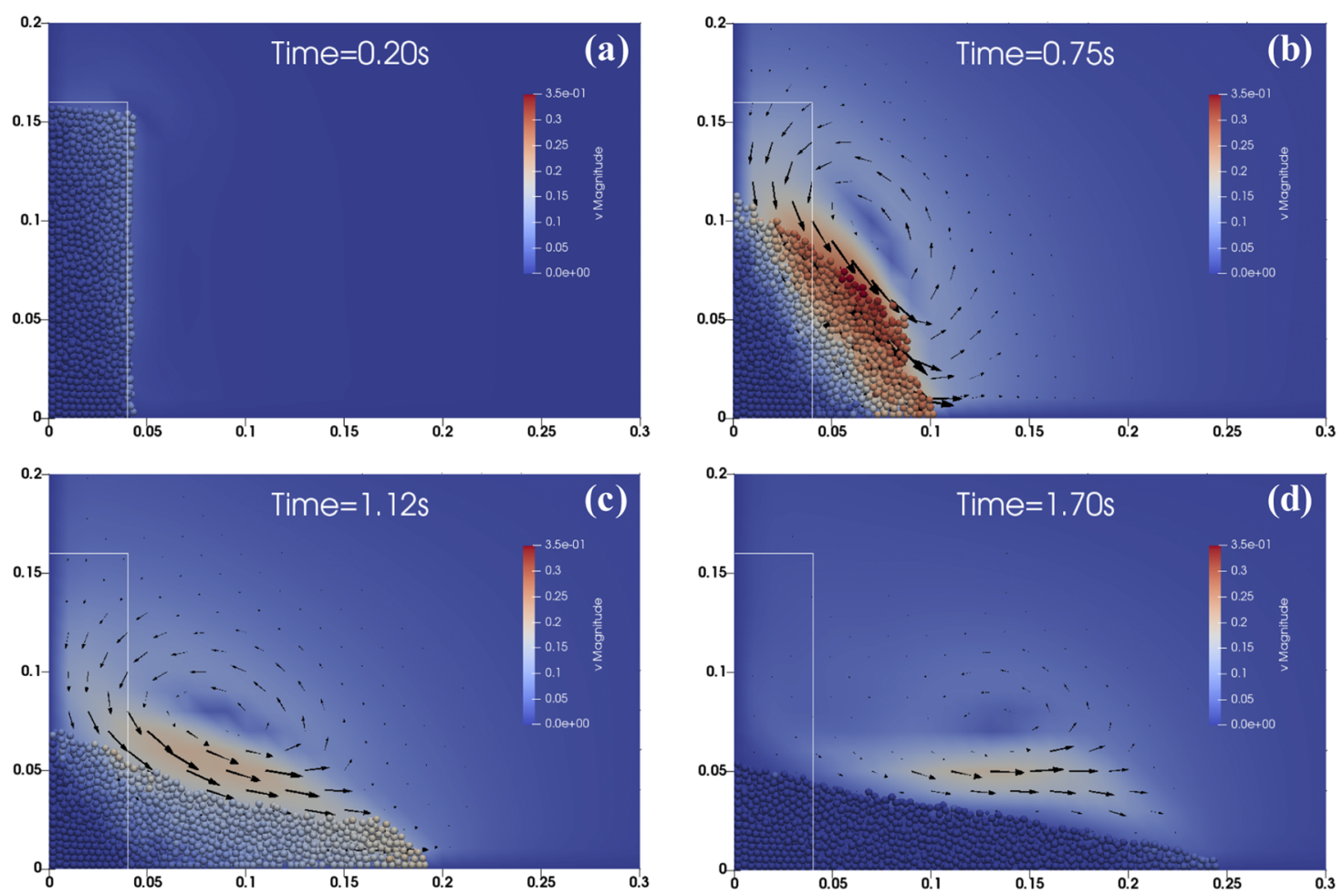

FIG. 7. Collapse sequences with $a=4$ at (a) $t=0.2 \mathrm{~s}$, initial stage; (b) $t=0.75 \mathrm{~s}$; (c) $t=1.12 \mathrm{~s}$; (d) $t=1.70 \mathrm{~s}$. Colors for both particles and fluid indicate the magnitude of velocity; arrows represent the velocity direction of fluid and their length indicates velocity magnitude. 


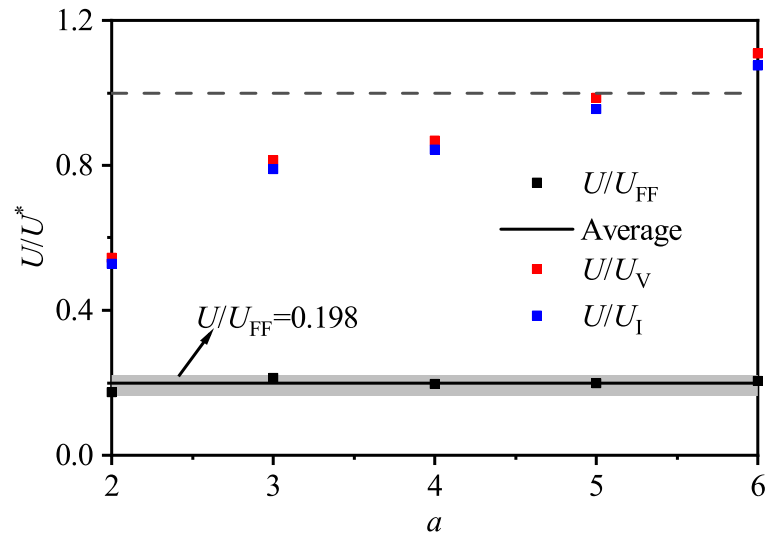

FIG. 8. Normalized propagation velocity using free-fall velocity $U_{\mathrm{FF}}$, viscous velocity $U_{V}$, and inertial velocity $U_{1}$. The gray area represents the error bar obtained from Fig. 11 of Bougouin and Lacaze.

Furthermore, the results normalized according to the viscous velocity $U_{\mathrm{V}}$ and inertial velocity $U_{\mathrm{I}}$ are also shown:

$$
U_{\mathrm{I}}=\sqrt{\frac{10}{3}\left(\frac{\rho_{\mathrm{p}}}{\rho_{\mathrm{f}}}-1\right) g d}, U_{\mathrm{V}}=\frac{\left(\rho_{\mathrm{p}}-\rho_{\mathrm{f}}\right) g d^{2}}{18 \mu_{\mathrm{f}}} .
$$

As shown in Fig. 8, normalization by $U_{\mathrm{V}}$ gives results that are closer to 1, providing further evidence that the flow considered in this study belongs to the viscous regime. Note that the so-called viscous inertial regime discussed by Bougouin and Lacaze ${ }^{32}$ is found to be the viscous regime according to their new definition considering column height. Additionally, the results in this section suggest that the coefficient $\zeta=U / U_{\mathrm{FF}}$ is likely to be independent of the density ratio $r$, at least from 1.37 to 1.6 .

\section{Runout distance}

The runout distance is one of the most important parameters in studies of granular collapse and natural disasters and is known to follow a power law relationship with respect to the initial aspect ratio. ${ }^{2,3,10,19,32}$ Recently, Yang et al. ${ }^{28}$ reported that the normalized final runout $\tilde{L}=\left(L_{\mathrm{f}}-L_{\mathrm{i}}\right) / L_{\mathrm{i}}$ and the densimetric Froude number

$$
\mathrm{Fr}_{\mathrm{d}}=\frac{V_{\mathrm{ft}}}{\sqrt{\left(\rho_{\mathrm{p}} / \rho_{\mathrm{f}}-1\right) g H_{\mathrm{ft}}}}
$$

were linearly related as $\tilde{L}=5.5 \mathrm{Fr}_{\mathrm{d}}$. According to their definition, the front particles are defined to be within $0.4 L_{\mathrm{i}}$ from the temporal leading runout, $V_{\mathrm{ft}}$ is the maximum front particles' average velocity during the collapse, and $H_{\mathrm{ft}}$ is the corresponding average height of that moment.

The simulated results for the correlation between $\tilde{L}$ and $\mathrm{Fr}_{\mathrm{d}}$ are shown in Fig. 9. It can be seen that the linear relationship holds for aspect ratios from 2 to 5 , whereas the deviations become significant when $a=6$. When the front particle reaches its maximum velocity, the front height $H_{\mathrm{ft}}$ increases too much in the case of $a=6$, that is obvious in the insets of Fig. 9, resulting in a decrease in $\mathrm{Fr}_{\mathrm{d}}$. This increase in height may be related to the much higher column, whereby particles are pushed forward by falling particles, leading to

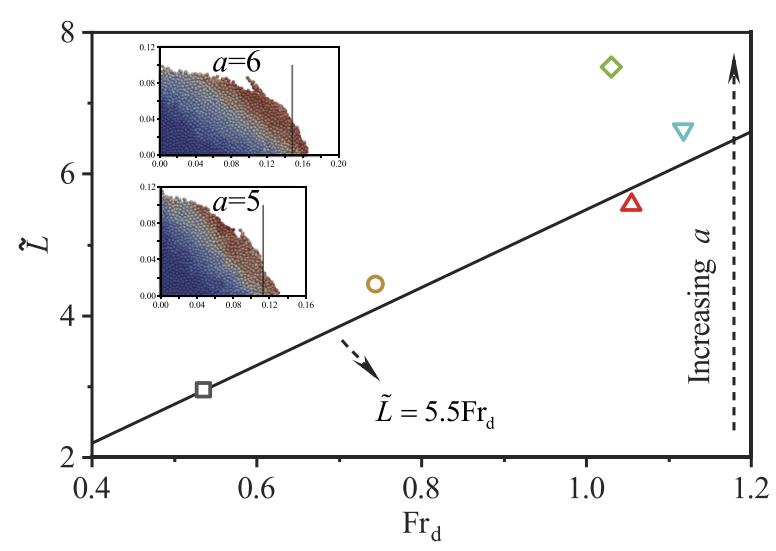

FIG. 9. Correlation between normalized final runout distance and densimetric Froude number. From bottom to top, the dots correspond to $a=2-6$. The vertical lines in the insets cut off the front particles.

a thicker front. Regardless of the mechanism, we emphasize that the original correlation focuses on the collapse of a low column ( $a$ $=0.8$ ), and our results show that the correlation holds for highcolumn collapses of up to $a=5$.

\section{E. Energy evolution}

\section{Overview of energy evolution}

In the granular collapse processes considered in this study, the only energy source is the particulate potential energy loss $\Delta E_{\mathrm{p}}=E_{\mathrm{p}}(t)$ - $E_{0}$, with $E_{0}=E_{\mathrm{p}}(0)$. During granular collapse, particles fall down, transforming their potential energy to particulate kinetic energy. The drag forces opposite to the particles' velocity retard the motion of the particles while dragging ambient fluid into motion. This process transfers kinetic energy from the particles to the fluid, calculated as $E_{\mathrm{k}}^{\mathrm{p}}=\sum m_{i}|\boldsymbol{u}|_{\mathrm{f}, i}^{2} / 2$ and $E_{\mathrm{f}}^{\mathrm{p}}=\sum \rho_{\mathrm{f}} \varepsilon_{\mathrm{f}, i} V_{i}|\boldsymbol{u}|_{\mathrm{f}, i}^{2} / 2$, respectively. Note that we ignore the rotational kinetic energy of the particles because this is negligible, with a maximum value of less than $1 \times 10^{-3} E_{0}$. The dissipated energy is mainly the result of fluid viscosity and particleparticle friction. Ignoring the elastic energy stored between particle contacts, this is given by $E_{\mathrm{d}}=\Delta E_{\mathrm{p}}-E_{\mathrm{k}}^{\mathrm{p}}-E_{\mathrm{f}}^{\mathrm{p}}$.

Figure 10 (a) presents the energy evolution during the collapse when $a=4$. It can be seen that about $77 \%$ of the particulate potential energy is devoted to the collapse, and most of this is dissipated. The maximum total kinetic energy of the fluid and particles only reaches $2.6 \%$ of $E_{0}$ and $2.0 \%$ of $E_{0}$, respectively.

We further analyze the partial kinetic energy, which is defined as $E_{\mathrm{k}, x}^{\mathrm{p}}=\sum m_{i} u_{\mathrm{f}, i}^{2} / 2, E_{\mathrm{k}, z}^{\mathrm{p}}=\sum m_{i} w_{\mathrm{f}, i}^{2} / 2$ and $E_{\mathrm{k}, x}^{\mathrm{f}}=\sum \rho_{\mathrm{f}} \varepsilon_{\mathrm{f}, i} V_{i} u_{\mathrm{f}, i}^{2} / 2$, $E_{\mathrm{k}, z}^{\mathrm{f}}=\sum \rho_{\mathrm{f}} \mathcal{E}_{\mathrm{f}, i} V_{i} w_{\mathrm{f}, i}^{2} / 2$ for the particles and fluid, respectively, where $u$ and $w$ are the $x$ - and $z$-components of velocity, respectively. The partial kinetic energy in the $y$-direction is ignored because it is insignificant, similar to the particles' rotational kinetic energy. Figure 10 (b) shows the partial kinetic energy evolution of both the fluid and particles when $a=4$. Note that the other groups give similar trends, so only the case of $a=4$ is presented here. Firstly, during the initial stages, a small energy bump appears (this will be discussed later). From $\sim 0.2$ to $0.4 \mathrm{~s}, E_{\mathrm{f}, z}^{\mathrm{p}}$ grows and $E_{\mathrm{k}, z}^{\mathrm{f}}$ becomes slightly lower, while 

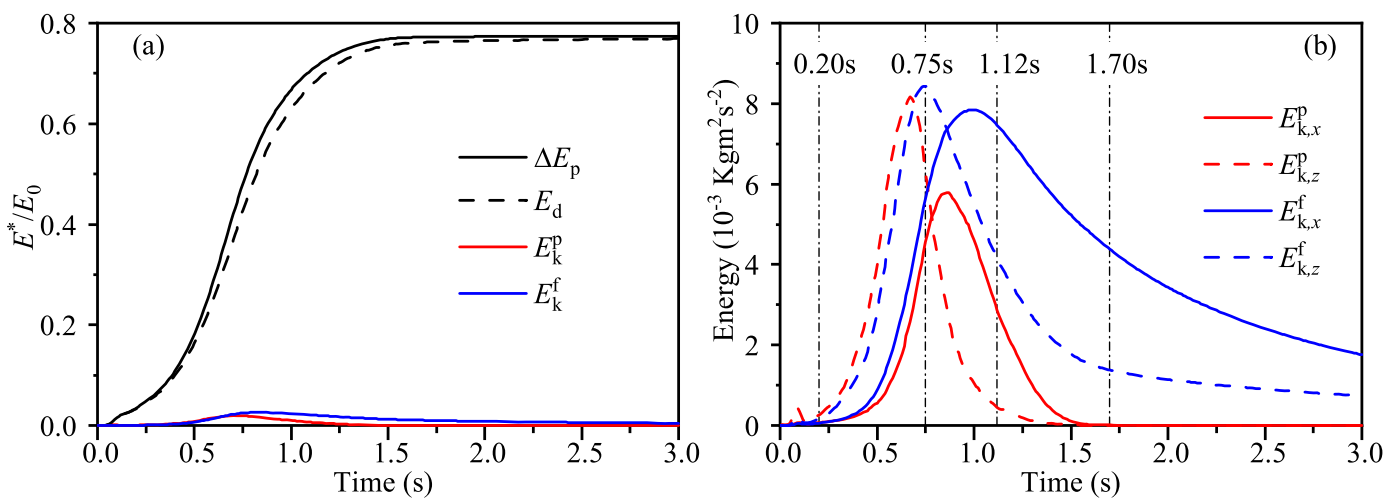

FIG. 10. Energy evolution in the case $a=4$. (a) Particulate potential energy loss and dissipated energy compared with total kinetic energy. (b) Partial kinetic energy. The dotted lines correspond to the four instants in Fig. 7. Note that the time interval between instants is $0.01 \mathrm{~s}$.

the $x$-components remain much lower. This indicates that motion along the $z$-direction is dominant in this stage. From then on, $E_{\mathrm{k} x}^{\mathrm{f}}$ starts to grow and $E_{\mathrm{k}, x}^{\mathrm{p}}$ follows, as a result of the formation of the large vortex. At $0.75 \mathrm{~s}$, both $E_{\mathrm{f}, z}^{\mathrm{p}}$ and $E_{\mathrm{k}, z}^{\mathrm{f}}$ have reached their peak values and the foot propagation moves into the constant velocity stage. From 0.75 to $1.12 \mathrm{~s}, E_{\mathrm{f}, z}^{\mathrm{p}}$ drops dramatically because the particles are moving vertically before hitting static particles in the corner, which form an inclined plane, and their motion changes to the $x$-direction, corresponding to the heap stage. ${ }^{23,29}$ The front particles are continually receiving energy from the turning particles and propagate at a constant velocity. Note that $E_{\mathrm{k}, x}^{\mathrm{f}}$ grows to be much larger than $E_{\mathrm{k}, x}^{\mathrm{p}}$ in the later stage of this period, and we believe that the positive drag of the fluid on the particles contributes to maintaining this constant velocity. After $1.12 \mathrm{~s}, \Delta E_{\mathrm{p}}$ stops increasing, $E_{\mathrm{f}, z}^{\mathrm{p}}$ almost vanishes, and $E_{\mathrm{k}, x}^{\mathrm{f}}$ starts to decrease, resulting in the particle motion decelerating. Almost all particles stop moving after $1.70 \mathrm{~s}$, and the fluid can barely drag any particles into motion, except for the leading particles. The collapse terminates and any existing fluid kinetic energy is gradually dissipated by viscosity.

\section{Discussion of the energy bump}

The small energy bump in the initial stage indicates that the granular column moves and soon decelerates, after which it continues to collapse. Note that we have applied a gate-removal process in previous cases, which may induce some elevation of the column. This process contributes to the bump, but is not completely responsible because a similar bump arises in the no-wall-removal case (gate unfixed instantly). In the following, we discuss the no-wall-removal case for brevity and clarity. We further investigate this stage in detail by reducing the output time interval to $0.0001 \mathrm{~s}$, as shown in Fig. 11. An unexpected energy crest for $E_{\mathrm{f}, x}^{\mathrm{p}}$ appears. To determine what happens during this stage, we simulate the collapse process with balanced gravity, $g^{\prime}=\rho_{\mathrm{f}} / \rho_{\mathrm{p}} g$, i.e., gravity balanced by buoyancy, and a similar bump appears, though the pile soon stops moving (see gray line in Fig. 11). Thus, it is clear that the horizontal movement is induced by the sudden release of the gate and the particles burst out when the inner force is relaxed. For completeness, the evolution of $E_{\mathrm{f}, x}^{\mathrm{p}}$ with the wall-removal stage is also presented. It can be seen that the energy peak is much smaller because the horizontal movement is confined.

Due to the horizontal motion of the particles, the vertical force chains that are initially holding the upper particles quickly dismiss [see Figs. 12(a) and 12(b)]. As a result, the particles fall down and the vertical kinetic energies increase. The force chains then start to rebuild and the particles decelerate under the inner friction and fluid viscosity as the kinetic energy gradually decreases. At around $0.08 \mathrm{~s}$, most of the particles have rearranged themselves to a temporary steady state and the kinetic energies tend to reach their minima. The particles in the right and upper parts of the domain continue to move under gravity, gradually eroding the inner force chains [see Figs. 12(d) and 12(e)], and the actual granular collapse begins at $0.1 \mathrm{~s}$.

Looking back at this procedure, the formation of the energy bump is mainly the result of particle destabilization and rearrangement. This study focuses on the collapse of high columns $\left(a_{\min }=2\right)$,

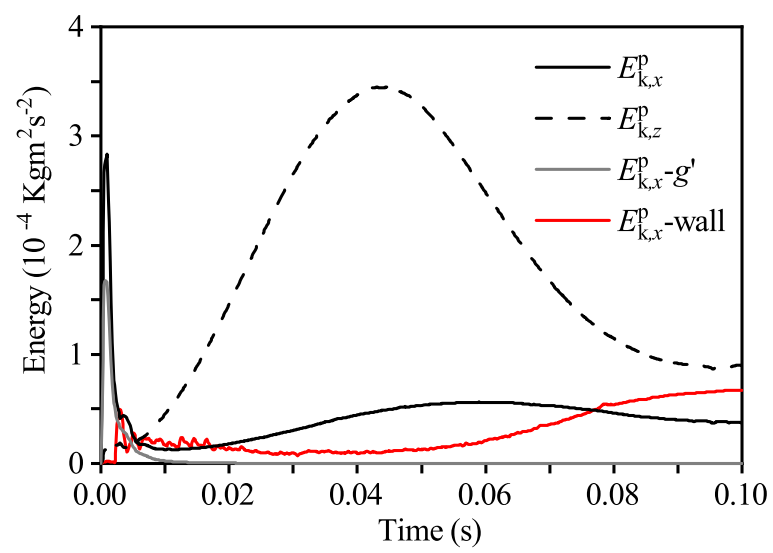

FIG. 11. Energy evolution during the initial stage in the case $a=4$ without wall removal. $E_{\mathrm{f}, x}^{\mathrm{p}}$ in the case $a=4$ with wall removal is shown for comparison. Note that the time interval between instants is $0.0001 \mathrm{~s}$. 
(a) $t=0 \mathrm{~s}$

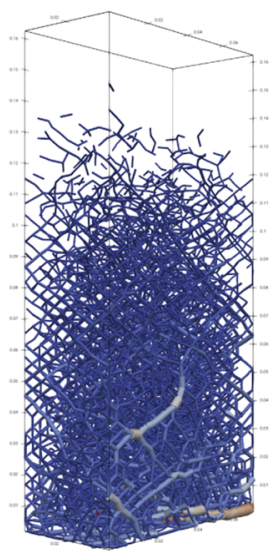

(b) $t=0.01 \mathrm{~s}$

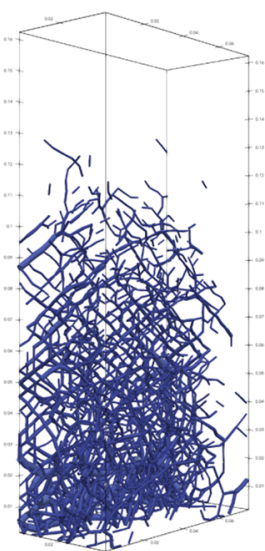

(c) $t=0.04 \mathrm{~s}$

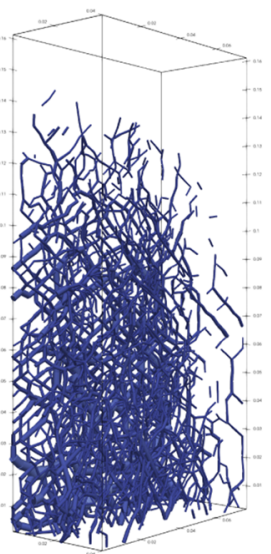

(d) $t=0.08 \mathrm{~s}$

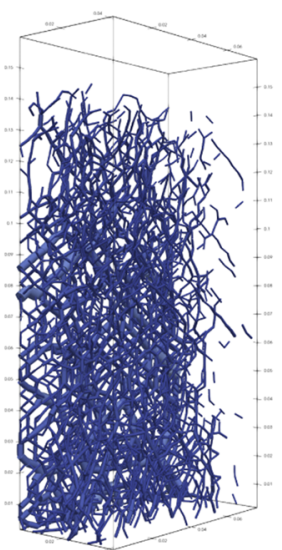

(e) $t=0.10 \mathrm{~s}$

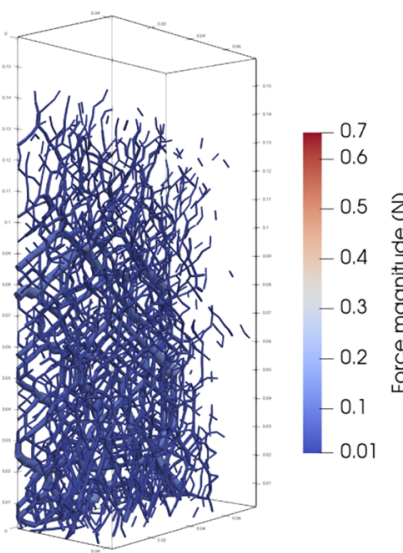

FIG. 12. Force chains during the initial stage in the case $a=4$ at different instants: (a) $t=0 \mathrm{~s}$, (b) $t=0.01 \mathrm{~s}$, (c) $t=0.04 \mathrm{~s}$ (d) $t=0.08 \mathrm{~s}$, (e) $t=0.10 \mathrm{~s}$. Note that only forces larger than $0.01 \mathrm{~N}$ are shown. The color and thickness correspond to the force magnitude.

which require longer falling durations, and the viscous regime collapse studied here exhibits trigger time that is quite long enough ${ }^{32}$ for the particles to rearrange and settle down. For shallow columns and other regimes, the procedure may be mixed with collapse and thus be barely distinguishable. ${ }^{28,29,31}$ This stage may have a considerable impact on the initial collapse. For example, when $a=4$, it takes $0.15 \mathrm{~s}$ for the vertical kinetic energy to reach its post-bump minimum with a wall and just $0.1 \mathrm{~s}$ without a wall. Thus, careful consideration is needed when analyzing the particle motions during the initial stage.

\section{CONCLUSIONS}

We have investigated the immersed granular collapse of high columns in a viscous regime through coupled CFD-DEM simulations. The input parameters were carefully determined from experiments, and the rolling friction coefficient, which is difficult to measure directly, was calibrated using a repose angle test. The correction of the granular parameters was verified through a dry granular collapse experiment, where we found that the wall-removal process was necessary for the reproduction of the collapse process, especially in the initial stage. With the well-determined parameters, the immersed granular collapse process was successfully reproduced, in terms of the front evolution and final deposit, by CFD-DEM simulations.

The normalized front evolutions were found to be similar for different aspect ratios and to exhibit three stages, i.e., acceleration, steady propagation, and deceleration. With the benefit of detailed particle-scale information from the CFD-DEM simulations, it was found that the constant velocity stage is maintained by the transition of particle motion from vertical to horizontal and the drag of the fluid. The constant propagation velocity is proportional to the free-fall velocity, and the coefficient matches well with that reported in a previous study. The linear correlation between the normalized final runout and the densimetric Froude number was reproduced in our high-column cases, despite it being originally proposed for shallow columns. Furthermore, a small energy bump observed during the initial stage was found to result from particle destabilization and rearrangement. A high column and long trigger time are necessary for the formation of this bump. Without either, this stage becomes mixed with the collapse process and can hardly be observed.

The CFD-DEM simulations described in this paper were validated based on experimental results and thus allow deeper investigations of the flow pattern of immersed granular flows. We have only focused on the viscous regime here, but future work will be devoted to different flow regimes and their individual collapse details.

\section{ACKNOWLEDGMENTS}

The authors are grateful for financial support from the National Natural Science Foundation of China (Grant Nos. 11872117 and 11432015). We thank Stuart Jenkinson, Ph.D., from Liwen Bianji, Edanz Group China (www.liwenbianji.cn/ac), for editing the English text of a draft of this manuscript.

\section{DATA AVAILABILITY}

The data that support the findings of this study are available from the corresponding author upon reasonable request.

\section{REFERENCES}

${ }^{1}$ S.-K. Hsu, J. Kuo, C.-L. Lo, C.-H. Tsai, W.-B. Doo, C.-Y. Ku, and J.-C. Sibuet, "Turbidity currents, submarine landslides and the 2006 Pingtung earthquake off SW Taiwan," Terr. Atmos. Ocean. Sci. 19(6), 767 (2008).

${ }^{2}$ G. Lube, H. E. Huppert, R. S. Sparks, and A. Freundt, "Collapses of twodimensional granular columns,” Phys. Rev. E 72(4), 041301 (2005).

${ }^{3}$ E. Lajeunesse, J. B. Monnier, and G. M. Homsy, "Granular slumping on a horizontal surface,” Phys. Fluids 17(10), 103302 (2005).

${ }^{4}$ N. J. Balmforth and R. R. Kerswell, "Granular collapse in two dimensions," J. Fluid Mech. 538, 399-428 (2005). 
${ }^{5}$ G. Lube, H. E. Huppert, R. S. J. Sparks, and M. A. Hallworth, "Axisymmetric collapses of granular columns," J. Fluid Mech. 508, 175-199 (2004).

${ }^{6}$ E. Lajeunesse, A. Mangeney-Castelnau, and J. P. Vilotte, "Spreading of a granular mass on a horizontal plane," Phys. Fluids 16(7), 2371-2381 (2004).

${ }^{7}$ E. L. Thompson and H. E. Huppert, "Granular column collapses: Further experimental results,” J. Fluid Mech. 575, 177-186 (2007).

${ }^{8} \mathrm{C}$. Meruane, A. Tamburrino, and O. Roche, "On the role of the ambient fluid on gravitational granular flow dynamics," J. Fluid Mech. 648, 381-404 (2010).

${ }^{9} \mathrm{C}$. Meruane, A. Tamburrino, and O. Roche, "Dynamics of dense granular flows of small-and-large-grain mixtures in an ambient fluid," Phys. Rev. E 86(2), 026311 (2012).

${ }^{10}$ L. Rondon, O. Pouliquen, and P. Aussillous, "Granular collapse in a fluid: Role of the initial volume fraction," Phys. Fluids 23(7), 073301 (2011).

${ }^{11}$ S. B. Savage, M. H. Babaei, and T. Dabros, "Modeling gravitational collapse of rectangular granular piles in air and water," Mech. Res. Commun. 56, 1-10 (2014).

${ }^{12}$ C. Wang, Y. Wang, C. Peng, and X. Meng, "Two-fluid smoothed particle hydrodynamics simulation of submerged granular column collapse," Mech. Res. Commun. 79, 15-23 (2017).

${ }^{13} \mathrm{C}$. Wang, Y. Wang, C. Peng, and X. Meng, "Dilatancy and compaction effects on the submerged granular column collapse," Phys. Fluids 29(10), 103307 (2017).

${ }^{14} \mathrm{M}$. Pailha, M. Nicolas, and O. Pouliquen, "Initiation of underwater granular avalanches: Influence of the initial volume fraction," Phys. Fluids 20(11), 111701 (2008).

${ }^{15} \mathrm{P}$. Si, H. Shi, and X. Yu, "Development of a mathematical model for submarine granular flows," Phys. Fluids 30(8), 083302 (2018).

${ }^{16} \mathrm{C}$ - $-\mathrm{H}$. Lee and Z. Huang, "A two-phase flow model for submarine granular flows: With an application to collapse of deeply-submerged granular columns," Adv. Water Resour. 115, 286-300 (2018).

${ }^{17}$ J. F. Richardson and W. N. Zaki, "Sedimentation and fluidisation: Part I," Chem. Eng. Res. Des. 75, S82-S100 (1997).

${ }^{18} \mathrm{~F}$. Engelund, On the Laminar and Turbulent Flows of Ground Water Through Homogeneous Sand (Akademiet for de Tekniske Videnskaber, 1953).

${ }^{19}$ C.-H. Lee, Z. Huang, and M.-L. Yu, "Collapse of submerged granular columns in loose packing: Experiment and two-phase flow simulation," Phys. Fluids 30(12), 123307 (2018)

${ }^{20} \mathrm{~V}$. Topin, F. Dubois, Y. Monerie, F. Perales, and A. Wachs, "Micro-rheology of dense particulate flows: Application to immersed avalanches," J. Non-Newtonian Fluid Mech. 166(1-2), 63-72 (2011).

${ }^{21} \mathrm{C}$. Cassar, M. Nicolas, and O. Pouliquen, "Submarine granular flows down inclined planes," Phys. Fluids 17(10), 103301 (2005).

${ }^{22}$ S. C. du Pont, P. Gondret, B. Perrin, and M. Rabaud, "Granular avalanches in fluids," Phys. Rev. Lett. 90(4), 044301 (2003).

${ }^{23}$ V. Topin, Y. Monerie, F. Perales, and F. Radjai, "Collapse dynamics and runout of dense granular materials in a fluid," Phys. Rev. Lett. 109(18), 188001 (2012).

${ }^{24} \mathrm{~K}$. Kumar, J.-Y. Delenne, and K. Soga, "Mechanics of granular column collapse in fluid at varying slope angles," J. Hydrodyn. 29(4), 529-541 (2017).

${ }^{25}$ E. Izard, L. Lacaze, T. Bonometti, and A. Pedrono, Numerical Modeling of a Granular Collapse Immersed in a Viscous Fluid (Springer-Verlag Singapore Pte Ltd., 2018).

${ }^{26}$ W.-J. Xu, X.-Y. Dong, and W.-T. Ding, "Analysis of fluid-particle interaction in granular materials using coupled SPH-DEM method," Powder Technol. 353, 459-472 (2019).

${ }^{27}$ G. C. Yang, L. Jing, C. Y. Kwok, and Y. D. Sobral, "A comprehensive parametric study of LBM-DEM for immersed granular flows," Comput. Geotech. 114, 103100 (2019).

${ }^{28}$ G. C. Yang, L. Jing, C. Y. Kwok, and Y. D. Sobral, "Pore-scale simulation of immersed granular collapse: Implications to submarine landslides," J. Geophys. Res. Earth Surf. 125(1), e2019JF005044 (2020).

${ }^{29}$ L. Jing, G. C. Yang, C. Y. Kwok, and Y. D. Sobral, "Dynamics and scaling laws of underwater granular collapse with varying aspect ratios," Phys. Rev. E 98(4), 042901 (2018).
${ }^{30}$ H. P. Zhu, Z. Y. Zhou, R. Y. Yang, and A. B. Yu, "Discrete particle simulation of particulate systems: Theoretical developments," Chem. Eng. Sci. 62(13), 33783396 (2007).

${ }^{31}$ L. Jing, G. C. Yang, C. Y. Kwok, and Y. D. Sobral, "Flow regimes and dynamic similarity of immersed granular collapse: A CFD-DEM investigation," Powder Technol. 345, 532-543 (2019).

${ }^{32}$ A. Bougouin and L. Lacaze, "Granular collapse in a fluid: Different flow regimes for an initially dense-packing," Phys. Rev. Fluids 3(6), 064305 (2018).

${ }^{33} \mathrm{Y}$. Tsuji, T. Tanaka, and T. Ishida, "Lagrangian numerical simulation of plug flow of cohesionless particles in a horizontal pipe," Powder Technol. 71(3), 239250 (1992).

${ }^{34}$ Y. Tsuji, T. Kawaguchi, and T. Tanaka, "Discrete particle simulation of twodimensional fluidized bed," Powder Technol. 77(1), 79-87 (1993).

${ }^{35}$ P. A. Cundall and O. D. L. Strack, "A discrete numerical model for granular assemblies," Geotechnique 29(1), 47-65 (1979).

${ }^{36}$ C. Kloss, C. Goniva, A. Hager, S. Amberger, and S. Pirker, "Models, algorithms and validation for opensource DEM and CFD-DEM," Prog. Comput. Fluid Dyn. 12(2-3), 140-152 (2012).

${ }^{37}$ Z. Y. Zhou, S. B. Kuang, K. W. Chu, and A. B. Yu, "Discrete particle simulation of particle-fluid flow: Model formulations and their applicability," J. Fluid Mech. 661, 482-510 (2010).

${ }^{38}$ A. Di Renzo and F. P. Di Maio, "Comparison of contact-force models for the simulation of collisions in DEM-based granular flow codes," Chem. Eng. Sci. 59(3), 525-541 (2004).

${ }^{39}$ W. R. A. Goossens, "Review of the empirical correlations for the drag coefficient of rigid spheres," Powder Technol. 352, 350-359 (2019).

${ }^{40}$ V. Agrawal, Y. Shinde, M. T. Shah, R. P. Utikar, V. K. Pareek, and J. B. Joshi, "Effect of drag models on CFD-DEM predictions of bubbling fluidized beds with Geldart D particles," Adv. Powder Technol. 29(11), 2658-2669 (2018).

${ }^{41}$ E. Biegert, B. Vowinckel, and E. Meiburg, "A collision model for grain-resolving simulations of flows over dense, mobile, polydisperse granular sediment beds," J. Comput. Phys. 340, 105-127 (2017).

${ }^{42}$ L. Girolami, V. Hergault, G. Vinay, and A. Wachs, "A three-dimensional discrete-grain model for the simulation of dam-break rectangular collapses: Comparison between numerical results and experiments," Granular Matter 14(3), 381-392 (2012).

${ }^{43}$ J. Ai, J.-F. Chen, J. M. Rotter, and J. Y. Ooi, "Assessment of rolling resistance models in discrete element simulations," Powder Technol. 206(3), 269-282 (2011).

${ }^{44}$ C. Goniva, C. Kloss, N. G. Deen, J. A. M. Kuipers, and S. Pirker, "Influence of rolling friction on single spout fluidized bed simulation," Particuology 10(5), 582-591 (2012).

${ }^{45}$ É. Guazzelli and O. Pouliquen, "Rheology of dense granular suspensions," J. Fluid Mech. 852, P1 (2018).

${ }^{46}$ Z. Yan, S. K. Wilkinson, E. H. Stitt, and M. Marigo, "Discrete element modelling (DEM) input parameters: Understanding their impact on model predictions using statistical analysis," Comput. Part. Mech. 2(3), 283-299 (2015).

${ }^{47}$ J. Frączek, A. Złobecki, and J. Zemanek, "Assessment of angle of repose of granular plant material using computer image analysis," J. Food Eng. 83(1), 17-22 (2007).

${ }^{48}$ H. T. Chou, C. F. Lee, Y. C. Chung, and S. S. Hsiau, "Discrete element modelling and experimental validation for the falling process of dry granular steps," Powder Technol. 231, 122-134 (2012).

${ }^{49} \mathrm{X}$. Xu, Q. Sun, F. Jin, and Y. Chen, "Measurements of velocity and pressure of a collapsing granular pile," Powder Technol. 303, 147-155 (2016).

${ }^{50} \mathrm{H}$. Wahyudi, K. Chu, and A. Yu, "3D particle-scale modeling of gas-solids flow and heat transfer in fluidized beds with an immersed tube," Int. J. Heat Mass Transfer 97, 521-537 (2016).

${ }^{51}$ R. Sun and H. Xiao, "SediFoam: A general-purpose, open-source CFD-DEM solver for particle-laden flow with emphasis on sediment transport," Comput. Geosci. 89, 207-219 (2016). 